\title{
APLICAÇÃO DAS TECNOLOGIAS IOT NO DESENVOLVIMENTO DE NOVOS PRODUTOS E SERVIÇOS
}

Caroline Gobbo Sá Cavalcante (carolinegobbosa@gmail.com.br) - Engenharia de Produção, Universidade Federal de Santa Catarina.

Diego de Castro Fettermann (dcfettermann@gmail.com) - Engenharia de Produção, Universidade Federal de Santa Catarina.

\section{RESUMO}

A Internet of Things (IoT) conduz a um novo paradigma tecnológico direcionado para a conexão entre coisas e pessoas em uma rede. Verifica-se que o estudo sobre o impacto que a aplicação das tecnologias IoT na gestão do desenvolvimento de produtos e serviços ainda é pouco abordado na literatura. A partir disso, tem-se por objetivo reunir da literatura as alterações sugeridas sobre o modelo tradicional para o desenvolvimento de produto que incorporem soluções IoT, para tanto foi realizada uma revisão sistemática de literatura. Os resultados deste estudo buscam identificar pontos de alteração dos processos de desenvolvimento de produtos para adaptar as atividades ao desenvolvimento de soluções IoT. As recomendações da literatura sobre o impacto das tecnologias IoT são organizadas de acordo com suas afinidades em cinco diferentes dimensões que afetam o desenvolvimento de produtos IoT: (i) cliente, (ii) produto/serviço, (iii) processamento, (iv) infraestrutura, $(v)$ habilidades. Verificou-se nos resultados, que a dimensão (i) cliente apresentou maior quantidade de recomendações da literatura, seguida da dimensão (ii) produto/serviço. Somente um artigo abordou a dimensão (v) habilidades, o que evidencia a incipiência do assunto e indica seu potencial de melhoria por meio da incorporação das recomendações contidas nestas dimensões.

Palavras-chave: Internet of Things, gestão de produtos e serviços, desenvolvimento de produto.

Área: Potencial da Internet of Things (IoT) e soluções de TIC para o desenvolvimento de produtos e serviços. 


\section{INTRODUÇÃO}

A Internet of Things (IoT) conduz a um novo paradigma tecnológico direcionado para a conexão de todos os tipos de dispositivos, denominados de "coisas", e pessoas por meio da internet (VERMESAN; FRIESS, 2014). Tem-se como objetivo trocar dados, otimizar processos ou monitorar dispositivos para gerar benefícios para os usuários (TARKOMA; AILISTO, 2013). A captura e o compartilhamento de informações na rede serão possíveis por meio de sensores, utilizados para detectar alterações físicas específicas; atuadores, que após serem ativados por sensores fazem conversão de energia; e protocolos de comunicação, que visam garantir a mesma linguagem de comunicação entre os dispositivos conectados (GUBBI et. al., 2013).

A entrada de dados ou ações dos usuários convergem no sentido de soluções conectadas e personalizadas, essência do processamento realizado remotamente com a utilização de banco de dados. A implementação das soluções com IoT passa a considerar, segundo a União Internacional de Telecomunicações (UIT) três pré-requisitos para uma solução IoT: (i) conectividade, (ii) sensores incorporados e (iii) inteligência incorporada (MEJTOFT, 2011). Com isso, as soluções IoT estão associadas a níveis cada vez maiores de conectividade; mais inteligência construída em dispositivos, objetos e sistemas; e consequentemente maior quantidade de dados gerados (VERMESAN, 2014). O valor agregado das soluções IoT supera conectar "coisas" e armazenar os dados, e assim incorporando a comunicação e o significado destes dados e seu processamento em benefício do usuário (NORONHA et al., 2014).

Estes dados dos produtos, "coisas" ainda podem ser combinadas com outros dados, adicionando mais conhecimento e valor para o usuário e para a empresa (PORTER; HEPPELMANN, 2015). Como exemplo no caso de uma indústria, em que os dispositivos conectados são capazes de comunicar entre si e se integrar com sistemas de estoque gerenciados por fornecedores, sistemas de suporte ao cliente, aplicativos de business intelligence e análise de negócios (LEE; LEE, 2015). Após o processamento, os dados podem ser utilizados para promover uma melhoria significativa no projeto do produto, processo de fabricação, redução de custos, escalabilidade, resiliência e sustentabilidade ambiental (BRADLEY et. al., 2017).

Tradicionalmente, as empresas enfatizavam a criação de valor através da oferta de produtos ou serviços de alta qualidade (HEMILÄ, 2015). A revisão de literatura sugeri o impacto que o IoT terá sobre eles, seja para uso doméstico, seja no uso voltado para empresas e negócios (ATZORI et al., 2010; MEJTOFT, 2011; HEMILÄ, 2015; HOLLER et al., 2016). Com a integração de tecnologias, os produtos de consumo tradicionais e bem estabelecidos podem ser transformados em produtos inteligentes e adquirirem habilidades de sensoriamento do ambiente, memória, processamento de dados, compartilhamento de informações, raciocínio e/ou atuação (KIRITSIS, 2011; DAWID et al., 2016).

Os produtos inteligentes podem ser classificados de acordo com o seu nível de maturidade em relação ao uso das tecnologias IoT (SÁ CAVALCANTE et al., 2017). As mudanças geradas por esses produtos vão além da cadeia de valor dentro de uma fábrica (PORTER; HEPPELMANN, 2015). Elas impactam toda a gestão do seu ciclo de vida, o qual abrange desde a concepção, fabricação, gestão da cadeia de abastecimento, manutenção e serviços, inclusive após a entrega do produto ao cliente, até seu destino final (KIRITSIS, 2011; TAO, 2016). Pode-se dizer que o IoT é um meio para alinhar os ciclos de vida físicos e de informação do produto (BRADLEY, 2017).

Os produtos inteligentes também possibilitam mudanças no relacionamento da empresa com o cliente em todas as etapas da cadeia de valor (TAO et al., 2016). Eles permitem o 
monitoramento contínuo de dados de desempenho real, a identificação e resolução de problemas de design que o teste não pôde expor, atualização de software, novos serviços (PORTER; HEPPELMANN, 2015). Tem-se como exemplo a atualização de software que viabiliza consertar um problema (PORTER; HEPPELMANN, 2015). Ao coletar dados sobre como os produtos se comportam e interagem, as empresas podem usá-los para entender e prever futuros comportamentos e melhorar experiências do usuário (UDEN et al., 2017).

Hoje o desenvolvimento de produtos físicos não reflete apenas a noção de objetos tangíveis, mas sim os de produtos individualizados com serviços associados, pois trazem maior valor ao cliente final (MEHRSAI et al., 2014; RYMASZEWSKA et al., 2017). Alguns estudiosos afirmam que as empresas de manufatura são simultaneamente empresas de serviços (BITNER, 1997; MATHIEU, 2001). A expectativa do consumidor em relação ao design, experiência de utilização e serviços mudou, por isso há uma necessidade de produtos rápidos, customizáveis e personalizados (MEHRSAI, 2014).

Há na literatura um enfoque na aplicação das tecnologias IoT em relação ao que é produzido fisicamente e nos seus benefícios. Por outro lado, pouco é abordado sobre a implicação dessas tecnologias em relação à forma como os produtos e serviços são desenvolvidos e às mudanças em toda a cadeia de valor da gestão de desenvolvimento desses (NAMBISAN, 2013). As informações relacionadas ao desenvolvimento encontram-se dispersas em vários artigos de IoT ou Indústria 4.0.

A partir das mudanças tecnológicas da conectividade nos produtos e serviços, incorporada pelo IoT, diversas adaptações precisam ser realizadas no gerenciamento de desenvolvimento de novos produtos. O desenvolvimento de um produto é compreendido a partir da realização de uma sequência de atividades, incluindo a aquisição de requisitos do cliente, a definição de especificações de projeto, a geração de conceitos de design, a seleção da arquitetura do produto, configuração e parâmetros-chave, criação de detalhes de design e recursos, distribuição, vendas e marketing, serviços e manutenção, reciclagem e descarte (LYU, 2017; ROZENFELD et al., 2006). Os modelos tradicionais de desenvolvimento de produto (ULRICH; EPPINGER, 2011; PAHL et al., 2005) estão restritos às atividades até o lançamento no mercado. Uma extensão desta proposta é a incorporação do acompanhamento do desempenho do produto no mercado, incluída no modelo de Rozenfeld et al. (2006). Apesar disso, as atividades previstas para o acompanhamento do produto no mercado desta proposta ainda estão restritas ao monitoramento do seu desempenho no processo de manufatura, assistência técnica, satisfação do cliente entre outros (ROZENFELD et al., 2006). A incorporação das possibilidades das tecnologias IoT nos produtos e serviços permitem uma transformação nas funcionalidades do produto em todo o seu ciclo de vida (KIRITSIS, 2011).

Apesar do grande potencial do IoT ainda é incipiente a utilização das tecnologias nos produtos e serviços sendo sua utilização uma necessidade das empresas (LEE; LEE, 2015). A incorporação do IoT resulta em alterações na pesquisa e no desenvolvimento, nos processos de fabricação, na gestão da cadeia de abastecimento, na manutenção e nos serviços, na abordagem do marketing, vendas e inclusive na forma de relacionamento entre a empresa e o cliente após a entrega do produto (DAWID, 2016; PORTER; HEPPELMANN, 2015; KIRITSIS, 2011). Também devem ser consideradas as competências necessárias para todas as etapas da cadeia de valor, novos conhecimentos como de processamento de informação, big data, algoritmos, softwares. O processamento de dados passará por mudanças, para melhor armazenamento e proteção dos dados (PORTER; HEPPELMANN, 2015).

A partir disso, este artigo tem por objetivo reunir da literatura as alterações sugeridas sobre o modelo tradicional para o desenvolvimento de produto que incorporem soluções IoT. Para tanto foi realizada uma revisão sistemática de literatura em duas bases de dados. Os resultados 
deste estudo buscam identificar pontos de alteração dos processos de desenvolvimento de produtos para adaptar as atividades ao desenvolvimento de soluções IoT.

Dessa forma, este artigo busca organizar as recomendações da literatura sobre as contribuições do IoT em cinco dimensões do desenvolvimento de produto e serviços traçadas pelos autores. Essa delimitação visa direcionar os futuros pesquisadores do tema e gestores da área.

\section{METODOLOGIA}

A revisão bibliográfica deu-se em duas plataformas de pesquisa conceituadas: Science direct e Scopus. Na pesquisa foram utilizadas as seguintes palavras chave agrupadas: ("IoT" OR "internet of things") AND ("smart product" OR "design product" OR "new product development"). Em seguidas verificou-se se constavam no resumo, nos títulos e nas palavras chave dos artigos encontrados. A partir dessa análise foram verificadas quarenta e sete citações na plataforma Scopus e apenas uma na Science direct. Após se descartar o que não era artigos, e os que estão repetidos, foi realizada a leitura dos resumos, títulos e palavras chave. Por fim, conseguiu-se aproveitar desse total apenas oito artigos (Tabela 1).

Tabela 1 - Revisão Bibliográfica

\begin{tabular}{|c|c|c|c|}
\hline Títulos & Periódico & Autores & $\begin{array}{c}\text { Citações } \\
\text { Google } \\
\text { Scholar }\end{array}$ \\
\hline $\begin{array}{l}\text { Click and drive Consumer attitude to product } \\
\text { development: Towards future transformations } \\
\text { of the driving experience }\end{array}$ & $\begin{array}{l}\text { Business Process } \\
\text { Management Journal }\end{array}$ & $\begin{array}{l}\text { Dominici et al. } \\
\qquad(2016)\end{array}$ & 4 \\
\hline $\begin{array}{c}\text { Make-to-XGrade for the Design and } \\
\text { Manufacturing of Flexible, Adaptive, and } \\
\text { Reactive Products }\end{array}$ & $\begin{array}{l}\text { 24th CIRP Design } \\
\text { Conference }\end{array}$ & $\begin{array}{l}\text { Mehrsai et al. } \\
\qquad(2014)\end{array}$ & 0 \\
\hline $\begin{array}{c}\text { Management science in the era of smart } \\
\text { consumer products: challenges and research } \\
\text { perspectives }\end{array}$ & $\begin{array}{c}\text { Central European } \\
\text { Journal of Operations } \\
\text { Research }\end{array}$ & Dawid et al. (2016) & 2 \\
\hline $\begin{array}{l}\text { Product modeling from knowledge, distributed } \\
\text { computing and lifecycle perspectives: A } \\
\text { literature review }\end{array}$ & Computers in Industry & $\begin{array}{l}\text { Lyu et al. } \\
\text { (2017) }\end{array}$ & 0 \\
\hline $\begin{array}{l}\text { How the Next Generation of Products Pushes to } \\
\text { Rethink the Role of Users and Designers }\end{array}$ & Procedia CIRP & $\begin{array}{l}\text { Montelisciani et al. } \\
\text { (2014) }\end{array}$ & 1 \\
\hline $\begin{array}{l}\text { Closed-loop PLM for intelligent products in the } \\
\text { era of the Internet of things }\end{array}$ & $\begin{array}{l}\text { Computer-Aided } \\
\text { Design }\end{array}$ & Kiritsis, D. (2011) & 262 \\
\hline $\begin{array}{c}\text { Internet of Things in product life-cycle energy } \\
\text { management }\end{array}$ & $\begin{array}{l}\text { Journal of Industrial } \\
\text { Information } \\
\text { Integration }\end{array}$ & $\begin{array}{l}\text { Tao et al. } \\
(2016)\end{array}$ & 17 \\
\hline $\begin{array}{l}\text { Internet of Things Capability and Alliance: } \\
\text { Entrepreneurial Orientation, Market Orientation } \\
\text { and Product and Process Innovation }\end{array}$ & Internet Research & $\begin{array}{l}\text { Jie et al. } \\
(2015)\end{array}$ & 10 \\
\hline
\end{tabular}

As recomendações da literatura sobre o impacto das tecnologias IoT no desenvolvimento de produtos e serviços foram organizadas de acordo com suas afinidades em cinco diferentes dimensões que afetam o desenvolvimento de produtos IoT. Na primeira dimensão, 
denominada (i) cliente, abordam-se os potenciais impactos no relacionamento da empresa com o mercado consumidor. A segunda dimensão, (ii) produto/serviço, refere-se aos itens e metodologias aplicadas no projeto do produto/serviço. A terceira dimensão, (iii) processamento, incorpora as recomendações para o processamento de informações e dados dos dispositivos IoT. A quarta dimensão, (iv) infraestrutura, refere-se aos requisitos físicos e de processos necessários para o pleno funcionamento dos produtos/serviços IoT. Por fim, a quinta dimensão, denominada (v) habilidades, está direcionada a identificar os conhecimentos técnicos e de gestão recomendados para o desenvolvimento de novos produtos e serviços IoT.

\section{RESULTADOS}

A análise bibliométrica foi realizada a partir de nove artigos. Deste total, 4 foram publicados em 2016, o que comprova quão atual é a temática. A publicação mais antiga entre os artigos data-se de 2011.

Há uma diversidade em relação às plataformas de publicação, principalmente no que tange as áreas, as quais variam entre negócios, design, engenharia, computação. Houve um predomínio de publicações de conferências CIPR, em que foram contabilizadas três publicações.

O artigo com maior número de citações na base de dados do Google Scholar é Kiritsis (2011), com 262 citações. Este artigo é também o mais antigo entre as publicações. Os segundo e terceiro mais citados nesta base são Tao et al. (2016) e o Jie et al. (2016), com 17 e 10 citações, respectivamente. Ambas publicações são de jornais voltados para área mais técnica, direcionada para as aplicações de internet nos processos produtivos.

Após a revisão, por conta da baixa quantidade de referências, foi incluso o artigo do Porter e Heppelmann (2015) para análise das dimensões do desenvolvimento de produto e serviços voltadas para o IoT, uma vez que este aborda extensamente a temática em questão no seu artigo. (Tabela 2). Sendo assim, a classificação será feita em cima de dez artigos.

Tabela 2 - Classificação dos artigos segundo das dimensões do desenvolvimento de produtos e serviços.

\begin{tabular}{|c|c|c|c|c|c|c|c|c|c|}
\hline Dimensões & 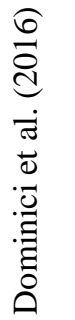 & 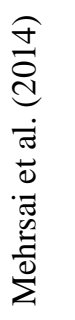 & 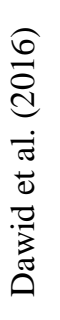 & 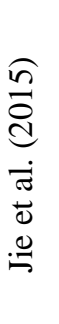 & 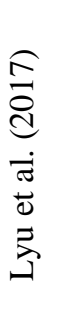 & 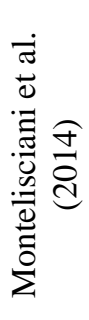 & 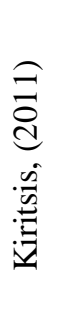 & 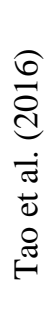 & 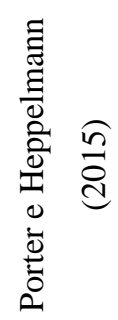 \\
\hline Cliente & $\mathrm{X}$ & $\mathrm{X}$ & $\mathrm{X}$ & $\mathrm{X}$ & $\mathrm{X}$ & $\mathrm{X}$ & $\mathrm{X}$ & $\mathrm{X}$ & $\mathrm{X}$ \\
\hline Produto/Serviço & & $\mathrm{X}$ & $\mathrm{X}$ & & $\mathrm{X}$ & $\mathrm{X}$ & $\mathrm{X}$ & $\mathrm{X}$ & $\mathrm{X}$ \\
\hline Processamento & & & & & & & $X$ & $\mathrm{X}$ & $\mathrm{X}$ \\
\hline Infraestrutura & & & $\mathrm{X}$ & & & & $\mathrm{X}$ & & $\mathrm{X}$ \\
\hline Habilidades & & & & & & & & & $\mathrm{X}$ \\
\hline
\end{tabular}

A análise das dimensões mostrou uma concentração dos artigos que apresentam mudanças relacionadas aos clientes após a implantação de tecnologias IoT. Todos os artigos abordaram a dimensão (i) cliente, seguido pela dimensão (ii) produto/serviço que foi trabalhada em sete artigos. Ambas as dimensões (iii) processamento e (iv) infraestrutura contaram com três propostas de artigos, e somente um artigo abordou a dimensão (v) habilidades. 
Verifica-se que o enfoque dos artigos na dimensão (i) cliente diz respeito às mudanças na relação com cliente após a aquisição do produto inteligente. A literatura menciona com frequência a possibilidade de acompanhamento de todas suas etapas do ciclo de vida, inclusive após a aquisição pelo cliente; os novos serviços a serem incorporados aos produtos; os upgrades serão feitos remotamente; os dados adquiridos pelos fabricantes para melhorias futuras. (MEHRSAI et al., 2014; DAWID et al.; 2016). A necessidade de incluir estas características associadas ao cliente é reforçada a partir de uma pesquisa sobre a influência de diferentes características do produto na intenção de compra do consumidor e da descoberta que a conectividade, a interatividade e a inteligência influenciam positivamente a intenção de compra por meio da experiência funcional (DAWID, 2016).

Foi verificado que a dimensão (ii) produtos/serviços é frequentemente abordada na literatura de forma integrada com a dimensão (i) cliente. É o caso de Mehrsai et al. (2014) que propôs uma estratégia de produção chamada MTX (Make-To-Xgrade), em que se tem um produto avatar (produto físico ou módulo com recursos e serviços virtuais), o qual faz uso de sensores e sistemas incorporados em sistemas físicos, com objetivo de oferecer uma experiência personalizada ao usuário. Esse conceito possibilita o acompanhamento do produto inteligente ao longo do seu ciclo de vida e sua relação com o usuário, o que permite mais funcionalidades, maior personalização, mudanças de configuração, oferecer serviços de manutenção preventiva para os clientes, e coleta de dados para os fabricantes (MEHRSAI et al., 2014). Ainda na dimensão (ii) produtos/serviços, Lyu, et al. (2017) apresenta os sistemas de modelagem de produtos baseados na web, que são desenvolvidos principalmente para dois propósitos: modelagem de produtos distribuídos e visualização de produtos. A modelagem de produtos distribuídos baseada na Web é considerada como uma extensão de dados de produtos e modelagem de conhecimento em sistemas centralizados para sistemas distribuídos usando tecnologias da web para suportar processos colaborativos de desenvolvimento (LYU et al., 2017).

Na dimensão (iv) infraestrutura, Dawid et al (2016) expõe as alterações no gerenciamento da cadeia de suprimentos resultantes do desenvolvimento de produtos IoT, que podem variar em relação às características produto e a forma proposta para o seu gerenciamento durante o ciclo de vida. Nesta proposta é ressaltada a importância da colaboração entre indústrias e fornecedores para desenvolver, construir e distribuir produtos inteligentes. A eficiência da cadeia de suprimentos pode aumentar se houver quebra de confidencialidade dos componentes dos produtos inteligentes, o que facilita a possibilita interfaces padronizadas de diferentes tecnologias. O gerenciamento da cadeia de suprimentos inteligente pode se beneficiar dos estudos mais recentes direcionados à ciência gerencial e a teoria dos jogos para analisar o projeto da cadeia de suprimentos, e assim adaptar modelos e métodos aplicados. Ainda é ressaltada a possibilidade de que produtos inteligentes podem exigir diferentes canais de distribuição em comparação com os tradicionais (DAWID, 2016).

Quanto à dimensão (v) habilidades, Porter e Heppelmann (2015) mencionam as mudanças provocadas pelos produtos inteligentes nas habilidades e na cultura empresarial. A empresa além de ser um fabricante de produtos tradicionais passa a ser uma empresa de software. Portanto, faz necessário contratar especialistas em engenharia de aplicativos, desenvolvimento de interface de usuário e integração de sistemas e, mais notavelmente, cientistas de dados capazes de construir e executar as análises automatizadas que ajudem a traduzir dados em ação, analistas de negócios. Além de treinar os vendedores para que estejam aptos a venderem os novos serviços.

Entre as propostas incorporadas na dimensão (iii) processamento, foi incluído a questão da segurança e o risco de ataques cibernéticos (PORTER; HEPPELMANN, 2015). Tao et al. (2016) traz soluções IoT para a área de gestão de dados da indústria de energia, como fusão 
de dados multi-sensores, reconhecimento difuso (fuzzy), computação em nuvem, computação ubíqua e mineração de dados (data mining), que podem ser usadas para realizar controle de consumo inteligente. A aquisição de dados ainda pode ser configurada de três modos de acordo com os diferentes objetos identificados. $\mathrm{Na}$ (i) aquisição estática, utiliza-se diferentes tipos de sensores de alta potência, RFID, sensores infravermelhos, escaneamento a laser, barra planar; para a (ii) aquisição dinâmica, utiliza-se rede de sensores e GPS; e para (iii) aquisição de informação em ambiente difuso, utiliza-se por exemplo imagens de vídeo (TAO et al., 2016).

\section{CONSIDERAÇÕES FINAIS}

Este artigo teve por objetivo reunir da literatura as alterações sugeridas sobre o modelo tradicional para o desenvolvimento de produto que incorporem soluções IoT. Para tanto, foi realizada uma revisão sistemática de literatura. Os resultados encontrados apresentam as diretrizes para adaptar o gerenciamento de desenvolvimento de produto tradicional para o desenvolvimento de produtos IoT. A dimensão (v) cliente, que incorpora o relacionamento com o mercado, tais como coleta de dados de uso dos produtos/serviços, monitoramento de comportamento do cliente, customização e personalização do produto, foi a que apresentou maior quantidade de recomendações da literatura. Seguidas pelas dimensões (ii) produto/serviço e (iv) infraestrutura, respectivamente. Houve três propostas para a dimensão (iii) processamento e uma sobre a dimensão (v) habilidade, o que evidencia a incipiência do assunto e o quanto ainda pode ser desenvolvido.

Entre as contribuições deste estudo, os gestores de desenvolvimento das empresas podem utilizar os resultados indicados neste artigo para iniciar a transformação do processo de desenvolvimento aplicado nas empresas para torná-los aptos para o desenvolvimento de soluções IoT.

Por fim, as recomendações de trabalhos futuros estão direcionadas ao levantamento de casos reais de aplicação de tecnologias IoT no desenvolvimento de produtos e serviços, para análise de contribuição prática. E posteriormente confrontar essas informações com as obtidas em levantamento bibliográfico. Como sugestão pode-se ampliar o leque de palavras-chave a fim de obter uma amostra maior de pesquisa do tema na área científica.

\section{REFERÊNCIAS}

ATZORI, L.; IERA, A.; MORABITO, G. The internet of things: A survey. Computer networks, v. 54, n.15, p.2787-2805, 2010.

BITNER, M. J. Services marketing: perspectives on service excellence. J. Retail, v.73, n.1, p.3-6, 1997.

BRADLEY, R.; JAWAHIRA, I. S.; MURREL, N.; WHITNEY, J. Parallel Design of a Product and Internet of Things (IoT) Architecture to Minimize the Cost of Utilizing Big Data (BD) for Sustainable Value Creation. The 24th CIRP Conference on Life Cycle Engineering, v61, p. 58-62, 2017.

DAWID, H.; DECKER, R.; HERMANN, T.; JAHNKE, H.; KLAT, W.; KÖNIG, R.; STUMMER, C. Management science in the era of smart consumer products: challenges and research perspectives, janeiro, 2016. 
DOMINICI, G., ROBLEK, V., ABBATE, T., TANI, M.. Click and drive: Consumer attitude to product development: Towards future transformations of the driving experience. Business process management journal, v. 22, n. 2, p. 420-434, 2016.

GUBBI, J.; BUYYA, R.; MARUSIC, S.; PALANISWAMI, M. Internet of Things (IoT): A vision, architectural elements, and future directions. Future generation computer systems, v.29, n.7, p.1645-1660, 2013.

HEMILÄ, J. Service Innovations based on Internet of Things in Industrial Context. The ISPIM Innovation Summit, Brisbane, Australia 6-9 dezembro, 2015. Australia.

JIE, Y. U., SUBRAMANIAN, N., NING, K., EDWARDS, D. Product delivery service provider selection and customer satisfaction in the era of internet of things: A Chinese eretailers' perspective. International Journal Production Economics, v. 159, p. 104-116, 2015.

KIRITSIS, D. Closed-loop PLM for intelligent products in the era of the Internet of things. Computer-Aided Design v.43, p.479-501, 2011.

LEE, I.; LEE, K. The Internet of Things (IoT): Applications, investments, and challenges for enterprises. Business Horizons, v.58, n.4, p.431-440, 2015.

LYU, G.; CHU, X.; XUE, D. Product modeling from knowledge, distributed computing and lifecycle perspectives: A literature review. Computers in Industry, v.84, p.1-13, 2017.

MATHIEU, V. Service strategies within the manufacturing sector: benefits, costs and partnership. International Journal of Service Industry Management, v.12, n.5, p.451-475, 2001.

MEJTOFT, T. Internet of Things and Co-creation of Value. IEEE International Conferences on Internet of Things, and Cyber, Physical and Social Computing, p. 672-677, 2011.

MEHRSAI, A.; HENRIKSEN, B.; ROSTAD, C. C.; HRIBERNIK, K. A.; THOBEN, K. Make-to-XGrade for the Design and Manufacturing of Flexible, Adaptive, and Reactive Products. Procedia CIRP 21, p.199-205, 2014.

MONTELISCIANI, G., MAZZEI, D., FANTONI, G. How the Next Generation of Products Pushes to Rethink the Role of Users and Designers. Procedia CIRP, v. 21, p. 93-98, 2014.

NAMBISAN, S., "Information Technology and Product/Service Innovation: A Brief Assessment and Some Suggestions for Future Research", Journal of the Association for Information Systems, v14, n.4, pp.215-226, 2013.

NORONHA, A.; MORIARTY, R.; O'CONNELL, K.; VILLA, N. Attaining IoT Value: How To Move from Connecting Things to Capturing Insights. Gain an Edge by Taking Analytics to the Edge. CISCO Report, 2014.

PAHL, G.; BEITZ, W.; FELDHUSEN, J.; GROTE, K. Projeto na Engenharia. Blücher, 2005. 407p.

PORTER, M. E.; HEPPELMANN, J. E. How Smart, Connected Products are Transforming Companies. Harvard Business Review, v.93, n.10, p.97-114, 2015.

PORTER, M. E.; HEPPELMANN, J. E. How smart, connected products are transforming competition. Harvard Business Review, v.92, n.11, p.64-88, 2014.

RYMASZEWSKA, A; HELO, P.; GUNASEKARAN, A. IoT powered servitization of manufacturing - an exploratory case study. International Journal of Production Economics, março, 2017. 
ROZENFELD, H. et al. Gestão de Desenvolvimento de Produtos: uma referência para a melhoria do processo. São Paulo: Saraiva, 2006. 542 p.

SÁ CAVAlCANTE, C. G., FETTERMANN, D. D. C., DE ALMEIDA, T. D., ZANDONAI, G. A. Oportunidades de novos produtos com a utilização de tecnologias IoT. 4a Congresso Internacional de Desenvolvimento da Engenharia Industrial, Joinville 30-31 maio, 1 junho, 2017, Brasil.

TAO, F.; WANG, Y.; ZUO, Y.; YANG, H.; ZHANG, M. Internet of Things in product lifecycle energy management. Journal of Industrial Information Integration, v.1, p.26-39, 2016.

TARKOMA, S. AILISTO, H. The internet of things program: the finnish perspective. IEEE Communications Magazine, março, 2013.

TARKOMA, S. AILISTO, H. The internet of things program: the finnish perspective. IEEE Communications Magazine, março, 2013.

UDEN, L., UDEN, L., HE, W., HE, W. (2017)," How the Internet of Things can help knowledge management: a case study from the automotive domain ". Journal of Knowledge Management, v. 21, n.1, p. 57-70, 2017.

ULRICH, K.; EPPINGER, S. Product Design and Development. A Resource for Students and Professionals in the Field of Product Design and Development. McGraw-Hill/Irwin, 2011, 432p.

VERMESAN, O.; FRIESS, P. Internet of Things Strategic Research Roadmap, IERC Cluster Book, caps.2-3, 2014. 